OPEN ACCESS

Edited by:

Alan J. Pearce,

La Trobe University, Australia

Reviewed by:

John Leddy,

University at Buffalo, United States

Carlos Hermano da Justa Pinheiro,

Unifanor Wyden, Brazil

${ }^{*}$ Correspondence:

J. Patrick Neary

patrick.neary@uregina.ca

Specialty section: This article was submitted to Health, a section of the journal Frontiers in Human Neuroscience

Received: 30 September 2019 Accepted: 23 January 2020

Published: 12 February 2020

Citation:

Neary JP, Dudé CM, Singh J, Len TK and Bhambhani YN (2020) Pre-frontal Cortex Oxygenation Changes During Aerobic Exercise in Elite Athletes Experiencing Sport-Related Concussion

Front. Hum. Neurosci. 14:35. doi: 10.3389/fnhum.2020.00035

\section{Pre-frontal Cortex Oxygenation Changes During Aerobic Exercise in Elite Athletes Experiencing Sport-Related Concussion}

\author{
J. Patrick Neary ${ }^{1}$, Carolynn M. Dudé2 ${ }^{2}$ Jyotpal Singh ${ }^{1}$, Trevor K. Len ${ }^{1}$ \\ and Yagesh N. Bhambhani ${ }^{3}$
}

${ }^{1}$ Faculty of Kinesiology and Health Studies, University of Regina, Regina, SK, Canada, ${ }^{2}$ Independent Researcher, Moncton, NB, Canada, ${ }^{3}$ Faculty of Rehabilitation Medicine, University of Alberta, Edmonton, AB, Canada

Aims: Recent research suggests that aerobic exercise can be performed safely within the first week following a concussion injury and that early initiation of exercise may speed recovery. To better understand the physiological changes during a concussion, we tested the hypothesis that mild-to-intense exercise testing can be performed within days immediately following injury, and can be used to discern differences between the concussed and normal healthy state. Thus, the purpose was to observe the cerebral hemodynamic responses to incremental exercise testing performed acutely post-concussion in high-performance athletes.

Methods: This study was a within- and between-experimental design, with seven male university ice hockey teams participating. A subgroup of five players acted as control subjects (CON) and was tested at the same time as the 14 concussed (mTBI) players on Day 2, 4, and 7 post-concussion. A 5-min resting baseline and 5-min exercise bouts of mild (EX1), moderate (EX2), and high (EX3) intensity exercise were performed on a cycle ergometer. Near-infrared spectroscopy was used to monitor pre-frontal cortex oxy-haemoglobin $\left(\mathrm{HbO}_{2}\right)$, deoxy-haemoglobin $(\mathrm{HHb})$, and total blood volume (tHb) changes.

Results: ANOVA compared differences between testing days and groups, and although large percentage changes in $\mathrm{HbO}_{2}(20-30 \%), \mathrm{HHb}(30-40 \%)$, and $\mathrm{tHb}(30-40 \%)$ were recorded, no significant $(\rho \leq 0.05)$ differences in cerebral hemodynamics occurred between mTBI vs. CON during aerobic exercise testing on any day post-injury. Furthermore, there was a linear relationship between exercise intensity vs. cerebral hemodynamics during testing for each day $\left(r^{2}=0.83-0.99\right)$.

Conclusion: These results demonstrate two novel findings: (1) mild-to-intense aerobic exercise testing can be performed safely as early as Day 2 post-concussion injury in a controlled laboratory environment; and (2) evidence-based objective measures such as 
cerebral hemodynamics can easily be collected using near-infrared spectroscopy (NIRS) to monitor physiological changes during the first-week post-injury. This research has important implications for monitoring physiological recovery post-injury and establishing new rehabilitation guidelines.

Keywords: concussion, exercise testing, near-infrared spectroscopy, pre-frontal cortex oxygenation, physiology, athletes

\section{INTRODUCTION}

During the past 5-10 years, there has been a growing interest in sport-related concussion due to recent deaths in high-performance athletes (Kelly, 2001; O'Connor et al., 2017). The Centers for Disease Control and Prevention, National Center for Injury Prevention and Control have reported that there is an estimated between 1.7 and 3.8 million concussions per year in the USA alone (Rutland-Brown et al., 2006; Daneshvar et al., 2011), with approximately $30 \%$ being sport-related (Cassidy et al., 2004; Langlois et al., 2006; Gaw and Zonfrillo, 2016). Globally, it is estimated that 64-74 million individuals experience traumatic brain injury annually (Dewan et al., 2018).

Recently there has been a focus on the management of the athlete post-concussion, including when it is safe for the athlete to return-to-learn and return-to-play so that the likelihood of re-injury is decreased (Aubry et al., 2002; Collins et al., 2002; McCrory et al., 2005, 2009, 2013, 2017; Ellis et al., 2016; Terwilliger et al., 2016). Traditionally, "prescribed rest” has been used to ensure the safety of the athlete (Moser et al., 2012; Elbin et al., 2014; Thomas et al., 2015; Buckley et al., 2016). Although recent consensus statements recommend that concussed athletes must participate in a graduated, medically supervised exercise protocol before returning-to-play (Aubry et al., 2002; McCrory et al., 2005, 2009, 2013, 2017), less information is available on whether aerobic exercise can be performed immediately postinjury, i.e., with the first $72 \mathrm{~h}$. However, mounting research is recommending that mild-to-moderate aerobic exercise can be performed safely within the days and weeks following injury (Leddy et al., 2016a, 2017, 2018; Bishop et al., 2017b; Dech et al., 2019), although most studies initiate exercise as a rehabilitation strategy after $\sim 21$ days post-injury (Dematteo et al., 2015; Gagnon et al., 2016; Morissette et al., 2019).

Most recently, exertional exercise testing protocols, such as the Buffalo Concussion Treadmill and Bike Test have proven to be safe and efficacious (Leddy et al., 2016a, 2018; Haider et al., 2019; Morissette et al., 2019) in evaluating the athletes post-concussion. These laboratory testing protocols have been designed with the intent to differentiate the type of concussion injury (i.e., physiologic, vestibulo-occular, cervicogenic; Ellis et al., 2014, 2015; Leddy et al., 2016a), and to discern the physiological mechanisms (cerebral autoregulation, cerebrovascular reactivity, neurovascular coupling; Ellis et al., 2015, 2016, 2018; Neary et al., 2019). This research has added to our understanding of the pathophysiological changes that occur following a concussion (Len et al., 2011, 2013; Giza and Hovda, 2014; Bishop and Neary, 2017; Bishop et al., 2017b; Worts et al., 2019), and has also provided a greater understanding of how we can apply exercise safely as a treatment strategy. Thus, it appears that evidence supports the therapeutic benefits of mildto-moderate intensity physical exercise (Kozlowski, 2014; Hinds et al., 2016; Leddy et al., 2016a,b, 2018) and that prolonged rest may be detrimental during acute recovery post-concussion (Elbin et al., 2014; Thomas et al., 2015; Buckley et al., 2016; Wells et al., 2016).

This evolving research that has examined the pathophysiology of concussion, includes monitoring cerebral blood flow velocity using transcranial Doppler (Len et al., 2011; Wright et al., 2018), cerebral blood-oxygen-level-dependent (BOLD) changes using functional magnetic resonance imaging (fMRI; Mutch et al., 2014, 2018), blood pressure (La Fountaine et al., 2016, 2019; Bishop et al., 2017b; Wright et al., 2018), heart rate variability (HRV; Gall et al., 2004; La Fountaine et al., 2009; Bishop et al., 2017a,b), cortical activation (Pearce et al., 2019), and cerebral hemodynamics using near-infrared spectroscopy (Urban et al., 2015; Bishop and Neary, 2017). In particular, near-infrared spectroscopy (NIRS) is an optical technique that has been validated to show quantitative changes in blood volume (an indirect measure of blood flow velocity) and oxygenation in cerebral tissue (Ferrari et al., 2004; Bhambhani et al., 2006). This measurement is taken at the level of capillaries, arterioles and venules in cerebral tissue (Belardinelli et al., 1995). By measuring the concentration of both the oxygenated $\left(\mathrm{HbO}_{2}\right)$ and deoxygenated haemoglobin $(\mathrm{HHb})$, changes in total blood volume (i.e., total haemoglobin; $\mathrm{tHb}=\mathrm{HHb}+\mathrm{HbO}_{2}$ ) can be determined and has been suggested as a surrogate for cerebral blood flow (Wolf et al., 2007).

NIRS has been used to monitor cerebral hemodynamics in patients following concussion (Kontos et al., 2014; Urban et al., 2015; Bishop and Neary, 2017), and TBI (Bhambhani et al., 2006). The neurocognitive research by Kontos et al. (2014) and Urban et al. (2015) suggests clinical implications of using pre-frontal cortex NIRS to assess brain cognition and connectivity. Furthermore, the pre-frontal cortex has been suggested as a major area to measure these hemodynamic changes during a hypercapnic challenge (Bishop and Neary, 2017), but no studies to our knowledge are available that used NIRS during incremental exercise when assessing acute concussion within 24-48 h following injury. Collectively, these studies suggest that the vascular involvement of the pre-frontal cortex is important during an acute concussion.

Because there is mounting research focused on both physiologic changes and the importance of exercise post-concussion (Worts et al., 2019), the focus of our research was to monitor the pathophysiological changes in athletes prospectively in the days following sport-related concussion 
in response to acute incremental aerobic exercise. It was hypothesised that exercise could be used safely to assess the physiological status of the concussed athlete and that there would be differences in the cerebral hemodynamic response between healthy control vs. mTBI subjects. This study used an evidence-based approach to discern differences between groups to contribute to our understanding and insight into the physiological response to exercise post-mTBI.

\section{METHODOLOGY}

\section{Participants and Research Ethics}

Players from seven university men's ice hockey teams in the Atlantic University Sport of the Canadian University Sport association served as potential participants for this study. All procedures were approved by the University Research Ethics Board. Before the beginning of the varsity ice hockey season, demographic information, pre-participation medical information and history, concussion history, the Physical Activity Readiness Questionnaire (PAR-Q), and written informed consent was collected from 120 (70.2\%) of a total 171 eligible players. A total of 14 players sustained a diagnosed concussion, and follow-up testing was performed on the injured players. The concussed (mTBI) athlete was assessed within $48 \mathrm{~h}$ of the injury (D2) and then prospectively on Day 4 or 5 (D4) and Day 6-9 (D7). Logistically, it was difficult to test exactly on the same days for all athletes because of their personal schedules, so the concussed athlete was tested on a day as close to the abovementioned days as possible. Specifically, players were tested on the following days: all subjects for $\mathbf{D} 2$ were tested on day 2; for D4, three subjects were tested on day 5 , and six subjects were tested on day 4; for D7, 2 subjects were tested on day 6 , one subjects tested day 7 , three subjects tested on day 8 , and three subject tested on day 9. Five control (CON) players (one player at each university testing location where concussions occurred) were matched line-mates that exercised at the same time intervals as the concussed player. CON (matching linemate) players were tested on the same day in an uninjured state, to alleviate any discrepancy in time of testing later in the season to control for changes in fitness level throughout the season. Table 1 shows the demographic information for each group.

For consistency, the medical personnel was required to use the consensus guidelines (McCrory et al., 2005) to detect and recognise a concussion. Clearance by the team physician or Certified Athletic Trainer or physical therapist was required for return-to-play.

TABLE 1 | Summary of descriptive data for Control $(N=5)$ and Concussed subjects $(N=14)$.

\begin{tabular}{lcc}
\hline Variable & Control & Concussed \\
\hline$N$ & 5 & 14 \\
Age (years) & $25.0 \pm 1.0$ & $22.80 \pm 1.2$ \\
Height $(\mathrm{m})$ & $1.79 \pm 0.1$ & $1.85 \pm 0.1$ \\
Body mass $(\mathrm{kg})$ & $81.68 \pm 8.3$ & $90.20 \pm 6.1$ \\
BMl $\left(\mathrm{kg} / \mathrm{m}^{2}\right)$ & $25.56 \pm 1.2$ & $26.47 \pm 1.2$ \\
\hline
\end{tabular}

Values are mean \pm standard deviation.

\section{Procedures and Study Design}

The same testing protocol (graded aerobic exercise protocol) was used for both the mTBI and CON athletes to assess pre-frontal cortex blood volume and oxygenation changes. Following orientation and verbal description of the testing protocol, a Polar S810 heart rate monitor and optical probes of the NIRS instrument were attached to the athlete. The same trained research assistant administered all testing for consistency of measurement. Once all equipment was connected, $5 \mathrm{~min}$ of resting baseline heart rate and pre-frontal cortex oxygenation parameters were taken simultaneously. To account for circadian rhythms, each post-concussion testing day for each mTBI athlete was scheduled at the same time of the day.

\section{Exercise Testing}

The exercise test lasted between 17 and $20 \mathrm{~min}$ and was performed on the same calibrated Monark $828 \mathrm{E}$ cycle ergometer (Monark Exercise, Vansbro, Sweden) throughout the study. The athlete began cycling at 40 Watts (pedaling frequency between 80-90 rpm) for a 2-min warm-up. Following the warm-up, a symptom-limited stepwise incremental exercise protocol began with each stage being $5 \mathrm{~min}$ in duration. The workload was increased to elicit heart rates which were mild (110-120 bpm, $\mathrm{EX}_{1}$ ), moderate (135-145 bpm, $\mathrm{EX}_{2}$ ) and intense (170-180 bpm, $\mathrm{EX}_{3}$ ), respectively. If the athlete felt they had to stop cycling, the protocol was terminated as recommended by current consensus guidelines (McCrory et al., 2005, 2009; Leddy et al., 2012). Control athletes completed an identical protocol on the same days as the injured athlete. The research assistant recorded the power output (Watts) during the first session for each athlete and this workload was repeated for all subsequent days.

\section{Near-Infrared Spectroscopy}

Using a spatially resolved oximeter (NIRO-300, Hamamatsu Photonics, Japan), quantitative changes in relative NIRS parameters $(\mu \mathrm{M})$ of $\mathrm{HbO}_{2}, \mathrm{HHb}$, and $\mathrm{tHb}$, were measured bilaterally over the frontal lobes. The NIRS unit consists of two channels for monitoring two separate regions of tissue simultaneously. Each channel has an emitting sensor probe that generates NIR light by four laser diodes via an optical fibre, and two silicone diodes contained within the detector probe that absorbs NIR light between the range of $760 \mathrm{~nm}$ and $950 \mathrm{~nm}$. The separation distance between the sensor and detector was $5 \mathrm{~cm}$, which allowed the light to penetrate to a depth of approximately $2.5 \mathrm{~cm}$ (Ferrari et al., 2004). The probes were placed bilaterally and equidistant from the midline, approximately $1 \mathrm{~cm}$ above the supraorbital ridge to avoid contamination of the signal by the temporalis muscle (Kleinschmidt et al., 1996; Obrig et al., 1996). The NIRS absorbency signal was averaged over 5-s intervals. The difference between peak values during the resting baseline values and the values during the test were calculated and used for analysis (Obrig et al., 1996). The reproducibility of NIRS has been documented (Mehagnoul-Schipper et al., 2002; Koike et al., 2004; Giacalone et al., 2017), and NIRS has been used previously in concussion research (Kontos et al., 2014; Urban et al., 2015; Bishop and Neary, 2017). A Polar S810 HR monitor (Polar 
TABLE 2 | The NIRS variables $\left(\mathrm{HbO}_{2}, \mathrm{HHb}, \mathrm{tHb} ; \mu \mathrm{M}\right)$ for each group, exercise condition and day of testing.

\begin{tabular}{lcccc}
\hline & GROUP & EX1 & EX2 & EX3 \\
\hline $\mathrm{HbO}_{2}(\mu \mathrm{M})$ & $\mathrm{CON}$ & $0.85 \pm 5.32$ & $8.54 \pm 7.08$ & $18.88 \pm 9.59$ \\
& $\mathrm{D} 2$ & $1.15 \pm 5.60$ & $11.36 \pm 10.02$ & $20.24 \pm 8.99$ \\
& $\mathrm{D} 4$ & $0.52 \pm 4.14$ & $9.14 \pm 8.18$ & $17.96 \pm 8.90$ \\
$\mathrm{HHb}(\mu \mathrm{M})$ & $\mathrm{D} 7$ & $-0.36 \pm 2.56$ & $9.14 \pm 8.14$ & $18.77 \pm 6.54$ \\
& $\mathrm{CON}$ & $2.09 \pm 2.68$ & $2.35 \pm 2.79$ & $4.46 \pm 2.75$ \\
& $\mathrm{D} 2$ & $2.64 \pm 2.87$ & $3.37 \pm 3.05$ & $5.53 \pm 4.31$ \\
$\mathrm{HHb}(\mu \mathrm{M})$ & $\mathrm{D} 4$ & $1.74 \pm 2.93$ & $2.31 \pm 2.40$ & $3.83 \pm 2.78$ \\
& $\mathrm{D} 7$ & $0.15 \pm 1.49$ & $1.40 \pm 2.24$ & $3.82 \pm 2.72$ \\
& $\mathrm{CON}$ & $2.42 \pm 6.81$ & $9.98 \pm 8.84$ & $22.36 \pm 11.54$ \\
& $\mathrm{D} 2$ & $3.40 \pm 4.68$ & $14.27 \pm 9.79$ & $24.60 \pm 8.51$ \\
& $\mathrm{D} 4$ & $4.04 \pm 7.19$ & $12.26 \pm 9.49$ & $21.92 \pm 11.10$ \\
& $\mathrm{D} 7$ & $1.96 \pm 6.26$ & $13.97 \pm 7.93$ & $25.12 \pm 1.90$
\end{tabular}

CON, control group; D2, day 2 post-injury; D4, day 4 post-injury; D7, day 7 postinjury. EX1, mild exercise; EX2, moderate exercise; EX3, intense exercise. Values are Mean \pm Standard Deviation.

Electro, Kempele, Finland) was used to record heart rate during the testing protocol.

\section{Data Analysis}

\section{Data Processing}

For descriptive purposes, the data were expressed as mean and standard deviation. All data were exported from the NIRO acquisition software at $6 \mathrm{~Hz}$, and processed in Microsoft Excel (Microsoft Office Home, Redmond, WA, USA). The acquisition software was equipped with tools to convert optical density signals to relative concentration units (micromolar; $\mu \mathrm{M}$ ) for oxyhaemoglobin $\left(\mathrm{HbO}_{2}\right)$, deoxyhaemoglobin $(\mathrm{HHb})$ and total haemoglobin $(\mathrm{tHb})$. The average and standard deviation of the entire 5-min exercise segments are presented in Table 2 and Figures 1-3. Recovery exercise data was not included in the analysis of plots because of missing data.

At Day 2 post-injury, one participant did not perform the third exercise segment. This resulted in five control participants completing the protocol $(n=4$ data sets for $\mathrm{HHb}$ due to signal contamination), six participants completing the protocol at Day 2 post-injury (with 1 participant not completing the third exercise load; $n=5$ data sets for $\mathrm{tHb}$ due to signal contamination),

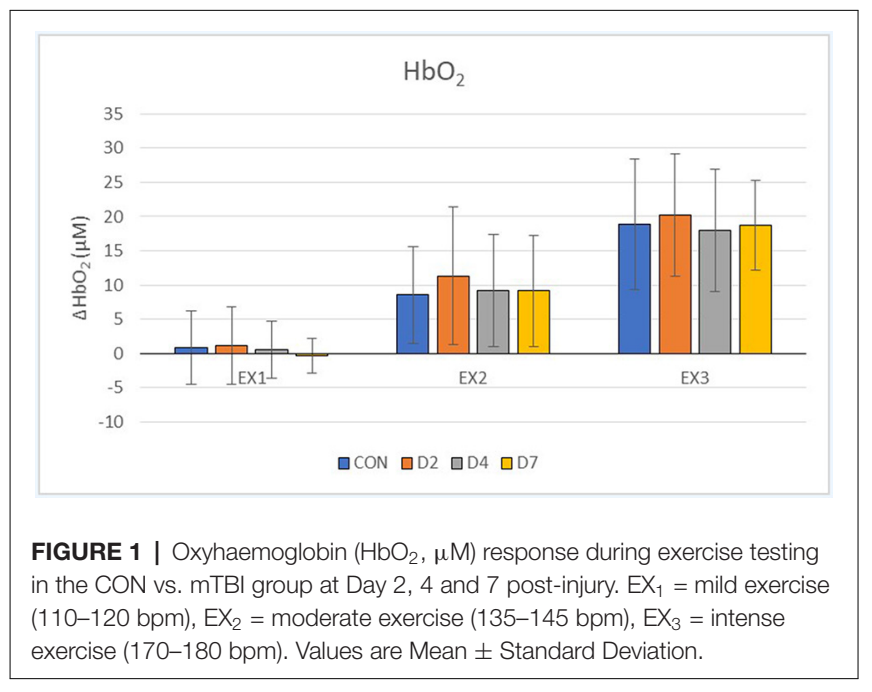

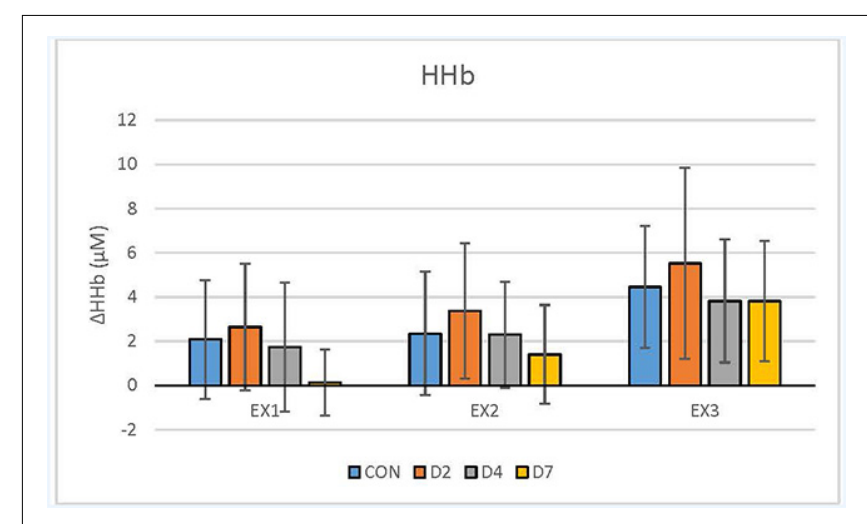

FIGURE 2 | De-oxyhaemoglobin ( $\mathrm{HHb}, \mu \mathrm{M})$ response during exercise testing in the CON vs. mTBI group at Day 2, 4 and 7 post-injury. $\mathrm{EX}_{1}=$ mild exercise (110-120 bpm), $\mathrm{EX}_{2}=$ moderate exercise (135-145 bpm), $\mathrm{EX}_{3}=$ intense exercise (170-180 bpm). Values are Mean \pm Standard Deviation.

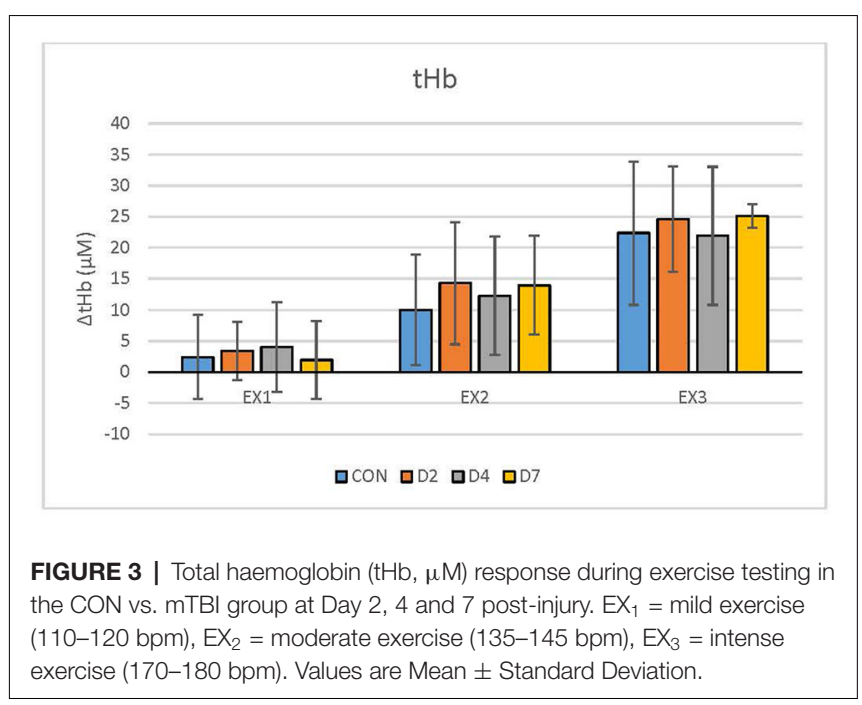

nine participants completing the protocol at day 4 post-injury ( $n=6$ for $\mathrm{HHb}$ and $n=8$ for $\mathrm{tHb}$ due to signal contamination), and 7 participants completing the protocol at Day 7 post-injury ( $n=4$ for $\mathrm{HHb}$ due to signal contamination).

\section{Statistical Analyses}

All NIRS data $\left(\mathrm{HbO}_{2}, \mathrm{HHb}\right.$ and $\left.\mathrm{tHb}\right)$ were assessed independently to observe changes between groups and conditions. One-way ANOVA's were used to determine that differences occurred between each condition [mild exercise $\left(\mathrm{EX}_{1}\right)$, moderate exercise $\left(\mathrm{EX}_{2}\right)$, intense exercise $\left.\left(\mathrm{EX}_{3}\right)\right]$ between controls and participants assessed the days post-concussion. Significance was set at $p \leq 0.05$. Due to some missing data points, an expectation maximation model with 25 iterations was used through SPSS (IBM Corp. Released 2019. IBM SPSS Statistics for Windows, Version 26.0. Armonk, NY, USA; IBM Corporation). Little's missing completely at random test (Little, 1988) was used to ensure that the data was indeed missing at random (with significance set at $p \leq 0.05$ ). 


\section{RESULTS}

\section{Descriptive Data}

The descriptive demographic data for the participants are presented in Table $\mathbf{1}$ and showed no differences between groups. Table 2 shows the NIRS variables for each group, exercise condition and day of testing. There were no statistically significant differences $(p<0.05)$ between the CON vs. mTBI group for any of the testing days post-injury (D2, D4, D7) for any of the oxygenation and hemodynamic (NIRS) variables (Table 2). There was a significant relationship between exercise intensity $\left(\mathrm{EX}_{1}, \mathrm{EX}_{2}, \mathrm{EX}_{3}\right)$ vs. the NIRS cerebral hemodynamic variables during exercise $\left(r^{2}=0.83-0.99\right)$ for all days of testing.

\section{DISCUSSION}

We demonstrated two important findings in this study: (1) that acute, short duration mild-to-intense exercise testing $(\mathrm{HR}=110 \mathrm{bpm}-170 \mathrm{bpm})$ can be performed safely in the days (D2, D4, D7) immediately following the diagnosis of concussion; and (2) that physiological measures can be easily collected and can be used to add to our understanding of the pathophysiological effects of concussion. This has important implications for treatment strategies, as current research suggests that sport-related concussion or mTBI is a physiological brain injury (McCrory et al., 2017; Leddy et al., 2018), and that concussion testing can potentially differentiate the type of mTBI (Ellis et al., 2015). Furthermore, our data support recent reports that physical exercise can be used safely when assessing recently concussed athletes within the first week post-injury in a controlled laboratory environment (Leddy et al., 2016a, 2017, 2018; Haider et al., 2019).

\section{Basis of Physiological Concussion Testing}

The primary objective of concussion testing is to characterise the physiologic response of the injured athlete. The effects of moderate-to-high intensity exercise on physiological function in the concussed state are unknown (Worts et al., 2019), and therefore we explored whether such exercise intensity could be safely maintained for a short duration (increments of 5-min workloads). To our knowledge this is the first study to systematically evaluate the physiological effects of repeated exercise tests using mild-to-intense exercise intensity (HR 110-170 bpm) performed within 2-days (D2) after the medical diagnosis of sport-related concussion in high-performance athletes. Based on the growing body of literature in this area, moderate-to-intense exercise testing can be performed in concussed athletes safely, but most studies were performed more than a week after injury (Baker et al., 2012; Ellis et al., 2015; Hinds et al., 2016; Leddy et al., 2016b, 2018; Haider et al., 2019; Morissette et al., 2019). Using an evidence-based approach, similar to our study, Leddy et al. (2011, 2017) developed the Buffalo Concussion Treadmill and Bike Tests to specifically evaluate symptom limitations in post-concussion syndrome (PCS) patients and showed that concussed athletes assessed on average of 33 weeks (6-36 weeks) post-injury could reliably differentiate physiological dysfunction and quantify exercise capacity in PCS patients. This test has now been used to assess the acute effects of acute concussion (Hinds et al., 2016; Haider et al., 2019). Furthermore, Ellis et al. (2015) conducted a review of the literature to describe and differentiate the type of concussion and suggested that concussed individuals can exercise to maximal volitional fatigue during supervised graded exercise testing. They classified the type of concussion as either physiologic, vestibulo-ocular, or cervicogenic (Ellis et al., 2015). It is well accepted that exercise increases the metabolic demand and stresses the autonomic nervous system function. Thus, dynamic cerebral autoregulation (Bailey et al., 2013), neurovascular coupling (Wright et al., 2017; Sharma et al., in press), heart rate (Haider et al., 2019) and HRV (Gall et al., 2004; La Fountaine et al., 2009), functional connectivity and brain activation (Kontos et al., 2014; Urban et al., 2015; Pearce et al., 2019) and blood pressure (La Fountaine et al., 2016, 2019) can be assessed to characterise the autonomic/physiological effects of acute mTBI. Therefore, this increasing body of literature demonstrates that objective physiologically based concussion testing using exercise is an important part of athlete management post-injury.

\section{Comparison of NIRS Hemodynamics Between Control vs. mTBI Participants}

Our results showed that athletes who sustained a sportrelated concussion or mTBI can undertake an incremental exercise test in 5-min stages from mild-to-intense exercise intensity on D2, D4 and D7 post-injury. Although we hypothesised that there would be significant differences between the CON and mTBI groups, the statistical analysis revealed no significant differences in the physiological responses in $\mathrm{HbO}_{2}, \mathrm{HHb}$ and $\mathrm{tHb}$ using NIRS, between groups or testing days (Figures 1-3). Previous research, including our own (Bishop and Neary, 2017), has shown that significant differences exist between days post-injury. For example, under resting conditions, $\mathrm{HbO}_{2}$ was statistically different $(p \leq 0.05)$ between days of recovery in mTBI participants; i.e., Day 1-3 vs. D7-14 and D4-6 vs. D7-14 (Bishop and Neary, 2017). As well, there were significant differences in the SD values between days post-injury, with the mTBI group showing reduced SD. However, it is important to note that this was in response to a hypercapnic (breath-hold) challenge which is a different paradigm than our exercise protocol. Hypercapnia has demonstrated that cerebral blood flow velocity is altered leading to cerebrovascular reactivity changes and differences between healthy controls and mTBI (Len et al., 2011, 2013; Mutch et al., 2014). Based on these observations, we extended our hypercapnic protocol (Neary et al., 2019) in the current study to include incremental $(5 \mathrm{~min})$ exercise workloads to assess the physiological consequences of mTBI in these athletes accustomed to intense physical exercise. No statistical differences in our hemodynamic data suggest that cerebral blood flow and oxygenation variables were not different during the exercise test in the days following acute concussion. When transitioning from rest to exercise, to sustain exercise intensity, energy (ATP) production is needed 
and was maintained adequately following concussion and did not compromise our athletes' ability to complete the testing protocol.

However, a word of caution is warranted, as our data showed that differences as much as $40 \%(p>0.05)$ exist between groups. Closer observation of our hemodynamic results showed that there were $30-40 \%$ differences in the mean value of $\mathrm{tHb}$ and $\mathrm{HHb}$ variables, and $20-30 \%$ differences in mean $\mathrm{HbO}_{2}$ between the CON vs. D2 and D4 at particular exercise workloads $\left(\mathrm{EX}_{1}\right.$, $\mathrm{EX}_{2}$; Figures 1-3). However, the standard deviations in our data were extremely large (2-5 times the mean) and eta values were low (0.01-0.02 for $\mathrm{HbO}_{2}, 0.06-0.12$ for $\mathrm{HHb}, 0.02-0.04$ for $\mathrm{tHb}$ ) making it difficult to reach statistical significance. Based on these statistical parameters, we have estimated that approximately 45 participants per group (CON, D2, D4 and D7) are required to demonstrate statistical differences. If the participants had baseline data collected first, a within-subject design based on this effect size will require 29 participants per group to demonstrate significance. This demonstrates the importance of including baseline testing in sport-related concussion research which has been documented previously (Len et al., 2013; Neary et al., 2019).

The high SD's are most likely related to two factors, the continuous wave NIRS device used in this study gives a relative change value and therefore differences between individual participants is common, and the small number of CON $(n=5)$ and mTBI $(n=14)$ participants in the study limited our statistical power. Individual differences could potentially nullify any differences that exist between groups as there is more variability to the data.

\section{Limitations of the Study}

As suggested above, the major limitation of our study was the small number of participants, especially in the CON group. Future studies of this nature must increase the number of control participants, both for within- and between-group comparisons. Because of the limited number of participants in this study, the standard deviation of the oxygenation variables were quite large. This is an inherent limitation of continuous-wave NIRS technology in that it gives a relative change value in oxygenation concentration $(\mu \mathrm{M})$. Typically, the best method to deal with relative differences between participants is to conduct a resting baseline and then zero the relative changes from the resting baseline (Bishop and Neary, 2017). We did this in our study to account for differences between participants but still, the SD values were high. Based on the SD and eta values we estimated that 45 subjects would be needed to show statistical significance, or based on a withinsubject design, 29 participants per group. Other limitations included not collecting rating of perceived exertion or symptom scores (Sport Concussion Assessment Tool; SCAT) to see how each athlete felt after their test, and whether this changed from D2 to D7. Anecdotally, the players reported feeling that exercise on D2 was difficult for them but felt better by D7 post-injury. However, all athletes were able to complete all the testing days. Recent research by Morissette et al. (2019) showed that symptomatic subjects when assessed at day 45 post-injury reported significantly higher ratings of perceived exertion vs. controls, although metabolic demands were not different at the same workload. The inclusion of a symptom checklist before and after the exercise testing may have provided additional information about their rating of perceived exertion, and considered for future research. However, the reader is reminded that SCAT scores are subjective and cannot always be relied upon. Finally, ECG recordings instead of using an HR monitor would have allowed us to calculate HRV changes from day-to-day. Previous research has demonstrated that HRV is altered in acute concussion (Gall et al., 2004; Papaioannou et al., 2008). Based on our results, and because of these limitations, it was not possible to determine the extent to which autonomic dysfunction occurred in our mTBI participants (Bishop et al., 2017b), or to characterise the type of concussion (Ellis et al., 2015).

\section{CONCLUSION}

This research demonstrated that a mild-to-intense incremental exercise test can be used safely within 2 days of injury, and can be repeated during the initial 7-9 days to assess brain physiology. This is the first study to demonstrate this finding in highly trained athletes with acute brain injury. Furthermore, regardless of whether statistical differences were apparent between groups or days of testing in this study, our results provide value physiological information on the status of the frontal lobe of the brain during the acute phase of sportrelated concussion.

\section{Future Directions and Implications}

We believe the future direction in concussion research is to continue to characterise the injured athlete physiologically and clinically following mTBI, and use the results to develop a physiologically based exercise prescription and treatment strategy that can be individualised to the athlete. Although we did not find statistically significant differences between the CON vs. mTBI group on days of testing, the physiological/clinical significance of the acute changes in the NIRS measurements indicates that concussed athletes are able to exercise intensely for short durations (5 $\mathrm{min}$ ) in the days following a concussion (days 2-7). These results suggest that the brain is still able to generate motor output to the muscles and cardiovascular system, despite having altered autonomic function (Len et al., 2011, 2013; Mutch et al., 2014). The practical implication of this is that clinicians can recommend short duration exercise as a viable alternative to try to enhance recovery, albeit, at mild-to-moderate intensity exercise appears more beneficial. Research by Haider et al. (2019) supports this contention and demonstrated that recently concussed ( $<10$ days) individuals could exercise safely at subthreshold heart rates. Furthermore, based on our results, it is our recommendation that mild-to-intense physical exercise testing must become part of the assessment protocol in contact sports where there is an increased risk and incidence for mTBI to occur, and testing of this nature must be included in future consensus guidelines. 


\section{DATA AVAILABILITY STATEMENT}

The datasets for this study can be found by contacting the corresponding author.

\section{ETHICS STATEMENT}

The studies involving human participants were reviewed and approved by University of New Brunswick Research Ethics Committee. The patients/participants provided their written informed consent to participate in this study.

\section{AUTHOR CONTRIBUTIONS}

Inception and design of the study were conceived by $\mathrm{CD}$ and JN. CD collected and analysed all data. JS and TL contributed

\section{REFERENCES}

Aubry, M., Cantu, R., Dvorak, J., Graf-Baumann, T., Johnston, K., Kelly, J., et al. (2002). Summary and agreement statement of the first international conference on concussion in sport, vienna 2001. Recommendations for the improvement of safety and health of athletes who may suffer concussive injuries. Br. J. Sports Med. 36, 6-10. doi: 10.1136/bjsm.36.1.6

Bailey, D. M., Jones, D. W., Sinnott, A., Brugniaux, J. V., New, K. J., Hodson, D., et al. (2013). Impaired cerebral haemodynamic function associated with chronic traumatic brain injury in professional boxers. Clin. Sci. 124, 177-189. doi: $10.1042 / \operatorname{cs} 20120259$

Baker, J. G., Freitas, M. S., Leddy, J. J., Kozlowski, K. F., and Willer, B. S. (2012). Return to full functioning after graded exercise assessment and progressive exercise treatment of postconcussion syndrome. Rehabil. Res. Pract. 2012:705309. doi: 10.1155/2012/705309

Belardinelli, R., Barstow, T. J., Porszasz, J., and Wasserman, K. (1995). Changes in skeletal muscle oxygenation during incremental exercise measured with near infrared spectroscopy. Eur. J. Appl. Physiol. Occup. Physiol. 70, 487-492. doi: $10.1007 /$ bf00634377

Bhambhani, Y., Maikala, R., Farag, M., and Rowland, G. (2006). Reliability of near-infrared spectroscopy measures of cerebral oxygenation and blood volume during handgrip exercise in nondisabled and traumatic braininjured subjects. J. Rehabil. Res. Dev. 43, 845-856. doi: 10.1682/jrrd.2005. 09.0151

Bishop, S. A., and Neary, J. P. (2017). Assessing prefrontal cortex oxygenation after sport concussion with near-infrared spectroscopy. Clin. Physiol. Funct. Imaging 38, 573-585. doi: 10.1111/cpf.12447

Bishop, S. A., Dech, R. T., Guzik, P., and Neary, J. P. (2017a). Heart rate variability and implication for sport concussion. Clin. Physiol. Funct. Imaging 38, 733-742. doi: $10.1111 /$ cpf.12487

Bishop, S. A., Dech, R., Baker, T., Butz, M., Aravinthan, K., and Neary, J. P. (2017b). Parasympathetic baroreflexes and heart rate variability during acute stage of sport concussion recovery. Brain Inj. 31, 247-259. doi: 10.1080/02699052.2016.1226385

Buckley, T. A., Munkasy, B. A., and Clouse, B. P. (2016). Acute cognitive and physical rest may not improve concussion recovery time. J. Head Trauma Rehabil. 31, 233-241. doi: 10.1097/htr.0000000000000165

Cassidy, J. D., Carroll, L. J., Peloso, P. M., Borg, J., von Holst, H., Holm, L., et al. (2004). Incidence, risk factors and prevention of mild traumatic brain injury: results of the WHO collaborating centre task force on mild traumatic brain injury. J. Rehabil. Med. 36, 28-60. doi: 10.1080/16501960410 023732

Collins, M., Lovell, M., Maroon, J., Cantu, R., and McKeag, D. (2002). Memory dysfunction eight days post-concussion in high school athletes. Med. Sci. Sport. Exerc. 34:S298. doi: 10.1097/00005768-20020500101681 to specific sections and assisted in the statistical analyses. All authors contributed to the writing and editing of the article.

\section{FUNDING}

We are grateful for the financial contribution from external research grants to conduct this research (Canadian Institutes of Health Research, CIHR \#84313).

\section{ACKNOWLEDGMENTS}

We would like to acknowledge the voluntary athletes that participated in this study. We would also like to thank Dr. Dave Goodman for his guidance and support in this research, and Dr. Michael Harrison for comments related to data collection.

Daneshvar, D. H., Nowinski, C. J., McKee, A. C., and Cantu, R. C. (2011) The epidemiology of sport-related concussion. Clin. Sports Med. 30, 1-17. doi: 10.1016/j.csm.2010.08.006

Dech, R. T., Bishop, S. A., and Neary, J. P. (2019). Why exercise may be beneficial in concussion rehabilitation: a cellular perspective. J. Sci. Med. Sport 22, 1090-1096. doi: 10.1016/j.jsams.2019.06.007

Dematteo, C., Volterman, K. A., Breithaupt, P. G., Claridge, E. A., Adamich, J., and Timmons, B. W. (2015). Exertion testing in youth with mild traumatic brain injury/concussion. Med. Sci. Sports Exerc. 47, 2283-2290. doi: 10.1249/MSS. 0000000000000682

Dewan, M. C., Rattani, A., Gupta, S., Baticulon, R. E., Hung, Y.-C., Punchak, M., et al. (2018). Estimating the global incidence of traumatic brain injury. J. Neurosurg., 1-18. doi: 10.3171/2017.10.JNS17352 [Epub ahead of print].

Elbin, R. J., Schatz, P., Lowder, H. B., and Kontos, A. P. (2014). An empirical review of treatment and rehabilitation approaches used in the acute, sub-acute and chronic phases of recovery following sports-related concussion. Curr. Treat Options Neurol. 16:320. doi: 10.1007/s11940-014-0320-7

Ellis, M. J., Figley, C. R., Mutch, W. A., Massicotte, E., Mikulis, D. J., Essig, M., et al. (2014). Neuroimaging in sports-related concussion management: current status and future directions. Curr. Res. Concussion 1, 33-39.

Ellis, M. J., Leddy, J. J., and Willer, B. (2015). Physiological, vestibuloocular and cervicogenic post-concussion disorders: an evidence-based classification system with directions for treatment. Brain Inj. 29, 238-248. doi: 10.3109/02699052.2014.965207

Ellis, M. J., Leddy, J., and Willer, B. (2016). Multi-disciplinary management of athletes with post-concussion syndrome: an evolving pathophysiological approach. Front. Neurol. 7:136. doi: 10.3389/fneur.2016.00136

Ellis, M. J., Leddy, J., Cordingley, D., and Willer, B. (2018). A physiological approach to assessment and rehabilitation of acute concussion in collegiate and professional athletes. Front. Neurol. 9:1115. doi: 10.3389/fneur.2018.01115

Ferrari, M., Mottola, L., and Quaresima, V. (2004). Principles, techniques and limitations of near infrared spectroscopy. Can. J. Appl. Physiol. 29, 463-487. doi: 10.1139/h04-031

Gagnon, I., Grilli, L., Friedman, D., and Iverson, G. L. (2016). A pilot study of active rehabilitation for adolescents who are slow to recover from sport-related concussion. Scand. J. Med. Sci. Sports 26, 299-306. doi: 10.1111/sms.12441

Gall, B., Parkhouse, W., and Goodman, D. (2004). Heart rate variability of recently concussed athletes at rest and exercise. Med. Sci. Sports Exerc. 36, 1269-1274 doi: 10.1249/01.mss.0000135787.73757.4d

Gaw, C. E., and Zonfrillo, M. R. (2016). Emergency department visits for head trauma in the United States. BMC Emerg. Med. 16:5. doi: 10.1186/s12873-0160071-8

Giacalone, G., Zanoletti, M., Contini, D., Re, R., Spinelli, L., Roveri, L., et al. (2017). Cerebral time domain-NIRS: reproducibility analysis, optical properties, haemoglobin species and tissue oxygen saturation in a cohort of adult subjects. Biomed. Opt. Express 8, 4987-5000. doi: 10.1364/boe.8.004987 
Giza, C. C., and Hovda, D. A. (2014). The new neurometabolic cascade of concussion. Neurosurgery 75, S24-S33. doi: 10.1227/neu.000000000 0000505

Haider, M. N., Leddy, J. J., Wilber, C. G., Viera, K. B., Bezherano, I., Wilkins, K. J., et al. (2019). The predictive capacity of the buffalo concussion treadmill test after sport-related concussion in adolescents. Front. Neurol. 10:395. doi: 10.3389/fneur.2019.00395

Hinds, A., Leddy, J., Freitas, M., Czuczman, N., and Willer, B. (2016). The effect of exertion on heart rate and rating of perceived exertion in acutely concussed individuals. J. Neurol. Neurophysiol. 7:388. doi: 10.4172/2155-9562. 1000388

Kelly, J. P. (2001). Loss of consciousness: pathophysiology and implications in grading and safe return to play. J. Athl. Train. 36, 249-252.

Kleinschmidt, A., Obrig, H., Requardt, M., Merboldt, K. D., Dirnagl, U., Villringer, A., et al. (1996). Simultaneous recording of cerebral blood oxygenation changes during human brain activation by magnetic resonance imaging and near-infrared spectroscopy. J. Cereb. Blood Flow Metab. 16, 817-826. doi: 10.1097/00004647-199609000-00006

Koike, A., Itoh, H., Oohara, R., Hoshimoto, M., Tajima, A., Aizawa, T., et al. (2004). Cerebral oxygenation during exercise in cardiac patients. Chest 125, 182-190. doi: 10.1378/chest.125.1.182

Kontos, A. P., Huppert, T. J., Beluk, N. H., Elbin, R. J., Henry, L. C., French, J., et al. (2014). Brain activation during neurocognitive testing using functional near-infrared spectroscopy in patients following concussion compared to healthy controls HHS public access. Brain Imaging Behav. 8, 621-634. doi: 10.1007/s11682-014-9289-9

Kozlowski, K. (2014). Exercise and concussion, part 2: exercise as a therapeutic intervention. Int. J. Athl. Ther. Train. 19, 28-32. doi: 10.1123/ijatt.2014-0007

La Fountaine, M. F., Heffernan, K. S., Gossett, J. D., Bauman, W. A., and De Meersman, R. E. (2009). Transient suppression of heart rate complexity in concussed athletes. Auton. Neurosci. 148, 101-103. doi: 10.1016/j.autneu.2009. 03.001

La Fountaine, M. F., Hohn, A. N., Testa, A. J., and Weir, J. P. (2019). Attenuation of spontaneous baroreceptor sensitivity after concussion. Med. Sci. Sports Exerc. 51, 792-797. doi: 10.1249/mss.0000000000001833

La Fountaine, M. F., Toda, M., Testa, A. J., and Hill-Lombardi, V. (2016). Autonomic nervous system responses to concussion: arterial pulse contour analysis. Front. Neurol. 7:13. doi: 10.3389/fneur.2016.00013

Langlois, J. A., Rutland-Brown, W., and Wald, M. M. (2006). The epidemiology and impact of traumatic brain injury: a brief overview. J. Head Trauma Rehabil. 21, 375-378. doi: 10.1097/00001199-200609000-00001

Leddy, J. J., Baker, J. G., Kozlowski, K., Bisson, L., and Willer, B. (2011). Reliability of a graded exercise test for assessing recovery from concussion. Clin. J. Sport Med. Off. J. Can. Acad. Sport Med. 21, 89-94. doi: 10.1097/jsm. 0b013e3181fdc721

Leddy, J. J., Haider, M. N., Ellis, M., and Willer, B. S. (2018). Exercise is medicine for concussion. Curr. Sports Med. Rep. 17, 262-270. doi: 10.1249/JSR. 0000000000000505

Leddy, J. J., Sandhu, H., Sodhi, V., Baker, J. G., and Willer, B. (2012). Rehabilitation of concussion and post-concussion syndrome. Sports Health 4, 147-154. doi: $10.1177 / 1941738111433673$

Leddy, J., Baker, J. G., Haider, M. N., Hinds, A., and Willer, B. (2017). A physiological approach to prolonged recovery from sport-related concussion. J. Athl. Train. 52, 299-308. doi: 10.4085/1062-6050-51.11.08

Leddy, J. J., Hinds, A., Sirica, D., and Willer, B. (2016a). The role of controlled exercise in concussion management. $P M R$ 8, S91-S100. doi: 10.1016/j.pmrj. 2015.10.017

Leddy, J. J., Baker, J. G., and Willer, B. (2016b). Active rehabilitation of concussion and post-concussion syndrome. Phys. Med. Rehabil. Clin. N. Am. 27, 437-454. doi: 10.1016/j.pmr.2015.12.003

Len, T. K., Neary, J. P., Asmundson, G. J. G., Candow, D. G., Goodman, D. G., Bjornson, B., et al. (2013). Serial monitoring of CO2 reactivity following sport concussion using hypocapnia and hypercapnia. Brain Inj. 27, 346-353. doi: 10.3109/02699052.2012.743185

Len, T. K., Neary, J. P., Asmundson, G. J. G., Goodman, D. G., Bjornson, B., and Bhambhani, Y. N. (2011). Cerebrovascular reactivity impairment after sportinduced concussion. Med. Sci. Sports Exerc. 43, 2241-2248. doi: 10.1249/mss. ob013e3182249539
Little, R. J. A. (1988). A test of missing completely at random for multivariate data with missing values. J. Am. Stat. Assoc. 83, 1198-1202. doi: 10.1080/01621459. 1988.10478722

McCrory, P., Johnston, K., Meeuwisse, W., Aubry, M., Cantu, R., Dvorak, J., et al. (2005). Summary and agreement statement of the 2 nd international conference on concussion in sport, prague 2004. Br. J. Sports Med. 39, 196-204. doi: 10.1136/bjsm.2005.018614

McCrory, P., Meeuwisse, W. H., Aubry, M., Cantu, B., Dvorak, J., Echemendia, R. J., et al. (2013). Consensus statement on concussion in sport: the 4th international conference on concussion in sport held in zurich, November 2012. Br. J. Sports Med. 47, 250-258. doi: 10.1136/bjsports-2013092313

McCrory, P., Meeuwisse, W., Dvorak, J., Aubry, M., Bailes, J., Broglio, S., et al. (2017). Consensus statement on concussion in sport-the 5th international conference on concussion in sport held in berlin, October 2016. Br. J. Sports Med. 51, 838-847. doi: 10.1136/bjsports-2017-097699

McCrory, P., Meeuwisse, W., Johnston, K., Dvorak, J., Aubry, M., Molloy, M., et al. (2009). Consensus statement on concussion in sport: the 3rd international conference on concussion in sport held in zurich, november 2008. J. Athl. Train. 44, 434-448. doi: 10.4085/1062-6050-44.4.434

Mehagnoul-Schipper, D. J., van der Kallen, B. F. W., Colier, W. N. J. M., van der Sluijs, M. C., van Erning, L. J. T. O., Thijssen, H. O. M., et al. (2002). Simultaneous measurements of cerebral oxygenation changes during brain activation by near-infrared spectroscopy and functional magnetic resonance imaging in healthy young and elderly subjects. Hum. Brain Mapp. 16, 14-23. doi: 10.1002/hbm.10026

Morissette, M. P., Cordingley, D. M., Ellis, M. J., and Leiter, J. R. S. (2019). Evaluation of early submaximal exercise tolerance in adolescents with symptomatic sport-related concussion. Med. Sci. Sports Exerc. doi: 10.1249/mss.0000000000002198 [Epub ahead of print].

Moser, R. S., Glatts, C., and Schatz, P. (2012). Efficacy of immediate and delayed cognitive and physical rest for treatment of sports-related concussion. J. Pediatr. 161, 922-926. doi: 10.1016/j.jpeds.2012.04.012

Mutch, W. A. C., Ellis, M. J., Graham, M. R., Wourms, V., Raban, R. Fisher, J. A., et al. (2014). Brain MRI CO2 stress testing: a pilot study in patients with concussion. PLoS One 9:e102181. doi: 10.1371/journal.pone. 0102181

Mutch, W. A. C., Ellis, M. J., Ryner, L. N., McDonald, P. J., Morissette, M. P., Pries, P., et al. (2018). Patient-specific alterations in co2 cerebrovascular responsiveness in acute and sub-acute sports-related concussion. Front. Neurol. 9:23. doi: 10.3389/fneur.2018.00023

Neary, J. P., Singh, J., Bishop, S. A., Dech, R. T., Butz, M. J. A., and Len, T. K. (2019) An evidence-based objective study protocol for evaluating cardiovascular and cerebrovascular indices following concussion: the neary protocol. Methods Protoc. 2:E23. doi: 10.3390/mps2010023

Obrig, H., Hirth, C., Junge-Hulsing, J. G., Doge, C., Wolf, T., Dirnagl, U., et al. (1996). Cerebral oxygenation changes in response to motor stimulation. J. Appl. Physiol. 81, 1174-1183. doi: 10.1152/jappl.1996.81.3.1174

O’Connor, K. L., Baker, M. M., Dalton, S. L., Dompier, T. P., Broglio, S. P., and Kerr, Z. Y. (2017). Epidemiology of sport-related concussions in high school athletes: national athletic treatment, injury and outcomes network (NATION), 2011-2012 through 2013-2014. J. Athl. Train. 52, 175-185. doi: 10.4085/10626050-52.1.15

Papaioannou, V., Giannakou, M., Maglaveras, N., Sofianos, E., and Giala, M. (2008). Investigation of heart rate and blood pressure variability, baroreflex sensitivity and approximate entropy in acute brain injury patients. J. Crit. Care 23, 380-386. doi: 10.1016/j.jcrc.2007.04.006

Pearce, A. J., Tommerdahl, M., and King, D. A. (2019). Neurophysiological abnormalities in individuals with persistent post-concussion symptoms. Neuroscience 408, 272-281. doi: 10.1016/j.neuroscience.2019. 04.019

Rutland-Brown, W., Langlois, J. A., Thomas, K. E., and Xi, Y. L. (2006). Incidence of traumatic brain injury in the United States, 2003. J. Head Trauma Rehabil. 21, 544-548. doi: 10.1097/00001199-200611000-00009

Sharma, A., Hind, K., Hume, P., Singh, J., and Neary, J. P. N. (in press). Neurovascular coupling by functional near infra-red spectroscopy and sportrelated concussion in retired rugby players: the UK rugby health project. Front. Hum. Neurosci. 14, 1-9. doi: 10.3389/fnhum.2020.00042 
Terwilliger, V. K., Pratson, L., Vaughan, C. G., and Gioia, G. A. (2016). Additional post-concussion impact exposure may affect recovery in adolescent athletes. J. Neurotrauma 33, 761-765. doi: 10.1089/neu.2015.4082

Thomas, D. G., Apps, J. N., Hoffmann, R. G., McCrea, M., and Hammeke, T. (2015). Benefits of strict rest after acute concussion: a randomized controlled trial. Pediatrics 135, 213-223. doi: 10.1542/peds. 2014-0966

Urban, K. J., Barlow, K. M., Jimenez, J. J., Goodyear, B. G., and Dunn, J. F. (2015). Functional near-infrared spectroscopy reveals reduced interhemispheric cortical communication after pediatric concussion. J. Neurotrauma 32, 833-840. doi: 10.1089/neu.2014.3577

Wells, E. M., Goodkin, H. P., and Griesbach, G. S. (2016). Challenges in determining the role of rest and exercise in the management of mild traumatic brain injury. J. Child Neurol. 31, 86-92. doi: 10.1177/0883073815570152

Wolf, M., Ferrari, M., and Quaresima, V. (2007). Progress of near-infrared spectroscopy and topography for brain and muscle clinical applications. J. Biomed. Opt. 12:62104. doi: 10.1117/1.2804899

Worts, P. R., Burkhart, S. O., and Kim, J.-S. (2019). A physiologically based approach to prescribing exercise following a sport-related concussion. Sports Med. 49, 683-706. doi: 10.1007/s40279-019-01065-1
Wright, A. D., Smirl, J. D., Bryk, K., and van Donkelaar, P. (2017). A prospective transcranial doppler ultrasound-based evaluation of the acute and cumulative effects of sport-related concussion on neurovascular coupling response dynamics. J. Neurotrauma 34, 3097-3106. doi: 10.1089/neu. 2017.5020

Wright, A. D., Smirl, J. D., Bryk, K., Fraser, S., Jakovac, M., and van Donkelaar, P. (2018). Sport-related concussion alters indices of dynamic cerebral autoregulation. Front. Neurol. 9:196. doi: 10.3389/fneur.2018.00196

Conflict of Interest: The authors declare that the research was conducted in the absence of any commercial or financial relationships that could be construed as a potential conflict of interest.

Copyright (c) 2020 Neary, Dudé, Singh, Len and Bhambhani. This is an open-access article distributed under the terms of the Creative Commons Attribution License (CC BY). The use, distribution or reproduction in other forums is permitted, provided the original author(s) and the copyright owner(s) are credited and that the original publication in this journal is cited, in accordance with accepted academic practice. No use, distribution or reproduction is permitted which does not comply with these terms. 